ca

the cell

division site is created in the grandparent, rather than the parent, of the daughter cell





In most bacteria, cell division occurs by binary fission, which is directed by FtsZ at the mid-cell. Alternatively, in Actinobacteria, cell division is polarized and is dependent on the DivIVA complex. However, very few studies have examined cell division outside of the context of model bacteria. Now, Abdelrahman et al. and Jutras, Scott et al. describe the biology of cell division in Chlamydia trachomatis and Borrelia burgdorferi, respectively, and reveal novel features of how cells divide in each of these important human pathogens.

Intriguingly, C. trachomatis lacks homologues of FtsZ or DivIVA, and so cell division must differ in some way from the mechanisms that have been described in other bacteria. Although some microscopy reports have suggested that $C$. trachomatis cells divide by binary fission, the mechanism has remained unclear, in part owing to the lack of amenability of obligate intracellular pathogens to laboratory study. Surprisingly, when Abdelrahman et al. used confocal microscopy to image C. trachomatis growth in HeLa cells, they found that cell division occurred at the cell oles. Cell growth prior to division was characterized by a polarized components, including the major outer membrane protein,

\title{
How pathogens divide
}

LPS and subdomains of the bacterial chromosome. The process of cell division was initiated by budding of the membrane at one of the poles and was asymmetric, in that the inheritance of cellular components was uneven between the mother cell and the newly formed daughter cell. However, by the time of cell division, which occurred at a septum that was formed during the budding process, the cellular content and sizes of the mother and daughter cells were similar to one another.

In contrast to $C$. trachomatis, the Lyme disease spirochete B. burgdorferi divides by FtsZ-dependent binary fission. However, little is known about cellular division in this species (or, indeed, in spirochetes more generally), in part owing to the challenge of studying slow-growing bacteria that have little genetic tractability. Jutras, Scott et al. used fluorescent D-alanine analogues to image peptidoglycan synthesis in B. burgdorferi, which showed that the elongation of the cell wall prior to cell division occurred in three discrete zones. Although elongation was concentrated at the mid-cell for most of the cell cycle, the two additional zones of elongation were observed shortly prior to cell division, which was concomitant with the initiation of nucleoid separation and septation. Interestingly, the locations of the additional elongation zones corresponded to the mid-cells of the daughter cells. Indeed, observation of the daughter cells following cell division showed that these zones remained active as mid-cell elongation zones, suggesting that, unusually, B. burgdorferi cells mark the sites at which the next generation will divide before dividing themselves. Observations of two other pathogens from the genus, Borrelia hermsii and Borrelia miyamotoi, revealed a similar elongation pattern; however, the mode of elongation did not seem to be conserved in other spirochete genera and may therefore be unique to Borrelia spp.

In each of the two studies, novel features of cell division were uncovered by investigating the full cell cycle. For C. trachomatis, daughter cells that resemble those produced by binary fission were found to instead be produced by budding from a pole. For B. burgdorferi, binary fission does occur, but the cell division site is created in the grandparent, rather than the parent, of the daughter cell.

Naomi Attar

ORIGINAL ARTICLES Abdelrahman, Y.et al. Polarized cell division of Chlamydia trachomatis. PLoS Pathog. 12, e1005822 (2016) | Jutras, B. L., Scott, M. et al. Lyme disease and relapsing fever Borrelia elongate through zones of peptidoglycan synthesis that mark division sites of daughter cells Proc. Natl Acad. Sci. USA 113, 9162-9170 (2016) FURTHER READING Haeusser, D. P. \& Margolin W. Splitsville: structural and functional insights into the dynamic bacterial Z ring. Nat. Rev. Microbiol. 14, 305-319 (2016) 